\title{
Anti-Acanthamoeba activity of contact lens solutions
}

Ingrid A Niszl, Miles B Markus

\begin{abstract}
Aims-This study was undertaken to investigate the effects of contact lens disinfecting solutions on strains of Acanthamoeba from the United Kingdom and southern Africa and to compare the results with those of other researchers. No information was previously available for southern African isolates.

Methods-11 contact lens solutions were tested on cysts of 10 strains of Acanthamoeba.

Results-Not all solutions used in the study were effective, with some for hard and gas permeable contact lenses being more satisfactory than those for soft contact lenses. The most effective of the gas permeable and hard contact lens solutions tested was Transoak $(0.01 \%$ (wt/vol) benzalkonium chloride), which killed cysts of all strains within 4 hours of exposure. Oxysept 1 (31 mg hydrogen peroxide/ml) was the best soft contact lens solution tested. It eliminated cysts of certain strains within 4 hours, whereas cysts of other strains were only inactivated within either 8 or 72 hours.

Conclusions-Manufacturers should be aware of the killing time for Acanthamoeba by contact lens solutions and should provide appropriate guidelines for the use thereof. The killing time for cysts of the African and UK isolates studied is, in general, similar. Therefore, it must in the present state of knowledge be assumed that usage guidelines suggested in the UK are also appropriate for travellers to South Africa and for local residents in South Africa.

(Br f Ophthalmol 1998;82:1033-1038)
\end{abstract}

Acanthamoeba keratitis among contact lens wearers is being reported with increasing frequency in various parts of the world. Evidence of the ease with which human infection can potentially occur is reflected by the isolation of Acanthamoeba from water drawn from bathroom taps and from dust around a washbasin. ${ }^{1}$ Domestic tap water has been implicated in a case of Acanthamoeba keratitis in the United Kingdom ${ }^{2}$ and this could also be a source of the disease in South Africa, where the organism occurs commonly in tap water and swimming pools. ${ }^{3}$ Since Acanthamoeba species are ubiquitous in the environment, lens care systems could possibly even become contaminated with cysts from the air. ${ }^{4}$ The presence in contact lens cases and solutions of bacteria originating from tap water or else- where predisposes to ocular Acanthamoeba infection. ${ }^{5-8}$ The amoebae feed on bacteria and multiply, with the result that large potential amoebic inocula may be present.

The time when the highly resistant cysts of Acanthamoeba are most likely to be killed appears to be while contact lenses are in storage/soaking and disinfecting solutions, the reason being that lenses are usually exposed to the active ingredients in these solutions for a minimum period of a few hours (usually overnight). Even though a patient may comply with general contact lens wear and care procedures recommended by lens manufacturers and healthcare professionals, a solution that does not kill Acanthamoeba may not protect the wearer against ocular infection with this organism. ${ }^{9}$ Experimental findings concerning the efficiency of chemical disinfection systems are inconclusive because the results vary. ${ }^{10-14}$ This could be due to variation in the susceptibility to contact lens solutions of different species and isolates of Acanthamoeba ${ }^{101215}$ or to the different experimental procedures which have been employed. ${ }^{12}{ }^{13}$ Standardised testing of the efficacy of contact lens disinfection systems against Acanthamoeba is, therefore, needed. ${ }^{16}$

The purpose of the present study was to compare the effectiveness of chemical storage/ soaking and disinfecting solutions on strains of Acanthamoeba isolated in southern Africa with reference strains from the United Kingdom. No such work has, to our knowledge, previously been carried out on any southern African isolate of the protozoon. Since the active ingredients of solutions available in southern Africa are similar to those used elsewhere in the world, a comparative study is of interest.

Use of solutions which are effective against a wide spectrum of Acanthamoeba strains would be desirable for contact lens wearers travelling abroad from a particular country, as well as those at home.

\section{Materials and methods}

ORGANISMS

The isolates which were used are listed in Table 1 , where references for three of the strains are given. Isoenzyme studies and/or restriction fragment length polymorphism analyses have been carried out on most of the strains for which there is, as yet, no information in the literature (cf Table 1), and will be published elsewhere. However, these results, based on biochemical data and morphological criteria for conventional specific classification, respectively, do not necessarily correlate-a phenomenon also observed by other researchers. ${ }^{19-21}$ 
Table 1 List of Acanthamoeba strains

\begin{tabular}{|c|c|c|c|c|}
\hline Isolate & $\begin{array}{l}\text { Environmental } \\
\text { source }\end{array}$ & Geographic origin & Date isolated & Designation \\
\hline $\mathrm{Ac} / \mathrm{PHL} / 23^{\star}$ & AK & England & & See Kilvington et al ${ }^{17}$ \\
\hline ATCC 30868 (CCAP 1501/2g) $\dagger$ & $\mathrm{AK}$ & England & 20 Sep 74 & ${\text { A } \text { castellanii }^{18}}$ \\
\hline ATCC 30873 (CCAP 1501/3d)† & $\mathrm{AK}$ & England & 10 May 74 & A polyphaga ${ }^{18}$ \\
\hline ATCC $50676 \ddagger$ & $\mathrm{AK}$ & Namibia or S Africa & $6 \mathrm{Jul} 90$ & \\
\hline ATCC $50677 \ddagger$ & $\mathrm{AK}$ & S Africa & 3 Aug 92 & \\
\hline ATCC $50678 \ddagger$ & $\mathrm{AK}$ & S Africa & 29 Dec 93 & \\
\hline ATCC $50679 \ddagger$ & $\mathrm{AK}$ & S Africa & 23 Mar 94 & \\
\hline ATCC $50680 \ddagger$ & $\mathrm{AK}$ & Botswana or S Africa & 9 Aug 94 & \\
\hline ATCC $50684 \ddagger$ & Contact lens & S Africa & $20 \mathrm{Jul} 93$ & \\
\hline ATCC $50686 \ddagger$ & Sewage sludge & S Africa & 26 Apr 87 & \\
\hline
\end{tabular}

$\star$ Donated by $\mathrm{S}$ Kilvington.

+Donated by DC Warhurst.

$\neq$ Records held by IA Niszl.

$\mathrm{AK}=$ Acanthamoeb $a$ keratitis.

Therefore, subgeneric taxonomic designations have not been attempted here for all the strains. With time and advances in knowledge, many specific identifications may in retrospect prove to be meaningless, particularly in such equivocal cases as are referred to above. However, our isolates have been deposited in the American Type Culture Collection (ATCC) so that they are readily available to other workers.

Cloning of Acanthamoeba was carried out as described previously for Mastigina $\mathrm{sp}^{22}$

Amoebae were axenically cultured at $30^{\circ} \mathrm{C}$ in antibiotic-free peptone-yeast extract glucose broth $^{22}$ in screw capped $80 \mathrm{~cm}^{2}(260 \mathrm{ml})$ Nunc tissue culture flasks. To obtain large numbers of organisms for experimentation, subcultures of Acanthamoeba trophozoites were grown in $500 \mathrm{ml}$ broth in 5 litre flasks on a shaker set at $100 \mathrm{rpm}$ for 42 hours.

Amoebic cysts were obtained by using trophozoites in constant $\mathrm{pH}$ encystment medium. ${ }^{23}$ Log phase axenic cultures of trophozoites (incubated at $30^{\circ} \mathrm{C}$ on a gyratory shaker set at $100 \mathrm{rpm}$ ) were centrifuged at 740 $\times g$ for 5 minutes and then handled according to the method of Davies et al. ${ }^{12}$ Incubation in encystment medium was at $30^{\circ} \mathrm{C}$ on a shaker set at $100 \mathrm{rpm}$ for 48 hours. After that time, more than $90 \%$ of cysts were mature, as determined by phase contrast microscopy. ${ }^{13}$ They were harvested and washed as described previously. ${ }^{22}$ Cysts were stored in fresh amoeba saline at $4^{\circ} \mathrm{C}$ and used within 7 days.

\section{CHEMICAL DISINFECTION}

Contact lens storage/soaking and disinfecting solutions (Table 2) were purchased from local retail stores or donated by the manufacturers. All solutions were used before their stated expiry date. Solutions were chosen so as to include a range of active ingredients for soft, hard, and gas permeable lenses (Table 2).

Tests were initially done on both cysts and trophozoites of strain ATCC 50676, using eight different contact lens solutions. Cysts were found to be more resistant to the solutions and because trophozoites which are kept in any solution for longer than approximately 24 hours will encyst, ${ }^{14}{ }^{24}$ it was decided to conduct subsequent experiments only on the cysts of the other strains which were studied. Eleven solutions were tested on all the strains (Fig 1, which does not include the results for Oxysept 1 Step because it is used for only 2 hours with a neutralising tablet).

Experiments were carried out in triplicate. A $1 \mathrm{ml}$ amount of each solution was dispensed aseptically into labelled $15 \mathrm{ml}$ plastic screw cap centrifuge tubes which could be used for spinning down directly at a later stage without the risk of losing trophozoites or cysts on transferring to a tube. Control tubes were prepared containing only amoeba saline and other controls with lens solutions alone. All tubes were left overnight to allow impregnation to take place. This is because certain chemicals have been shown to interact with storage containers by a surface adsorption process, ${ }^{25}$ which could possibly dilute the amount of active ingredient present. Our overnight impregnation with each solution was an attempt to deal with this problem. Tubes were kept in the dark during impregnation, and for the duration of the experiment, to prevent possible deactivation of solutions in light and to simulate conditions in a lens case.

Following impregnation and immediately before commencement of the experiment, solutions and amoeba saline were pipetted off and $1 \mathrm{ml}$ of fresh fluid was added to each tube. A $10 \mu \mathrm{l}$ cyst suspension containing approximately $1 \times 10^{4}$ cysts as has been recommended, ${ }^{26}$ was pipetted into each tube. Cyst counts were done on an Adams haemocytometer. The subsequent procedure was as described for cysts, ${ }^{22}$ except that the experimental times were different (Fig 1). In the case of Oxysept 1 Step, the neutralising tablet (5200 U catalase per tablet) was added at the same time as the solution and left in the tube for the duration of the disinfection period, according to the manufacturer's instructions.

After the last centrifugation, the material was plated out as previously explained. ${ }^{22}$ Plates were incubated at $30^{\circ} \mathrm{C}$ for 14 days and were examined daily for Acanthamoeba trophozoites by means of an inverted microscope. We assessed viability of organisms by their ability (or otherwise) to excyst and multiply, compared with control plates.

\section{STATISTICAL ANALYSIS}

A comparison test run on a SAS Version 6.1 computer package was used for statistical analysis. To evaluate differences between the responses of the various Acanthamoeba strains 


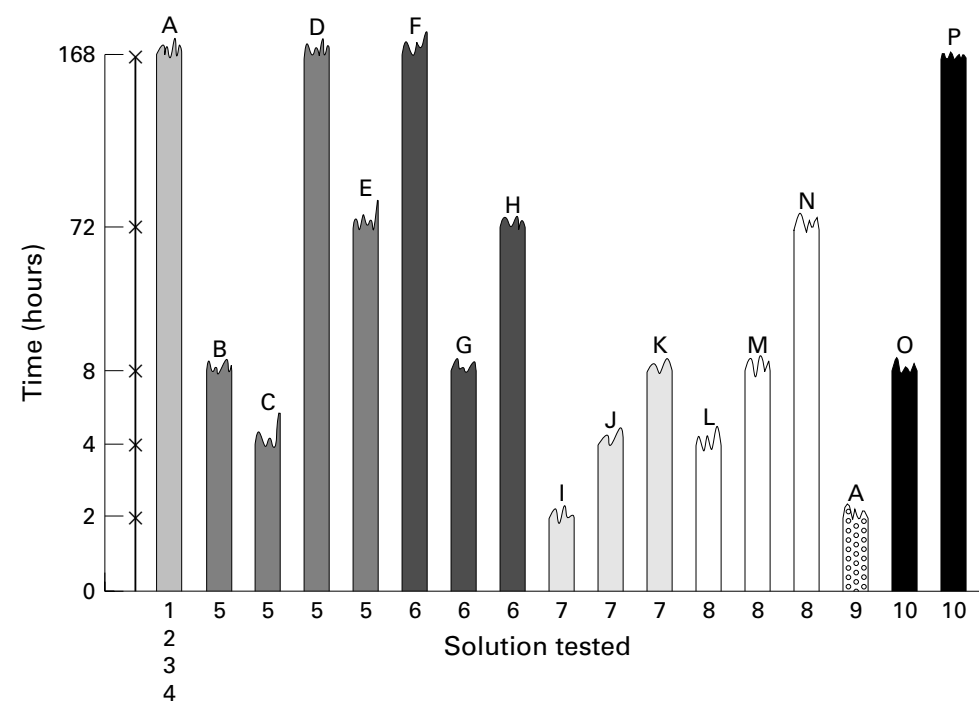

Figure 1 Viability of cysts of 10 strains of Acanthamoeba after exposure to contact lens disinfecting solutions. All 10 strains of Acanthamoeba were tested against each solution. The bars represent the variation in time to complete elimination of the cysts by different solutions. The jagged edges at the tops of the bars indicate that cysts were still viable at that time but not at the next testing time. Each bar is the result of three repeated experiments. Each experiment gave reproducible results. Key: $X=$ exposure time (hours) of cysts to solution; $A=$ all 10 strains of $A$ canthamoeba; $B=A c / P H L / 23, A T C C$ 50677, ATCC 50678, ATCC 50679, ATCC 50680; $C=$ ATCC 30868, ATCC 50676; $D=A T C C$ 50684; $E=A T C C$ 30873, $A T C C$ 50686; $F=A T C C$ 30873, $A T C C$ 50676, $A T C C$ 50679, ATCC 50684; $G=A c / P H L / 23, A T C C ~ 30868, A T C C$ 50678, ATCC 50686; $H=$ ATCC 50677, ATCC 50680; I = ATCC 30873, ATCC 50677, ATCC 50679, ATCC 50686; $\mathcal{F}=A c / P H L / 23, A T C C$ 30868, ATCC 50676, $A T C C$ 50680, $A T C C$ 50684; $K=$ ATCC 50678; $L=A T C C$ 30868, ATCC 50679; $M=A c / P H L / 23, A T C C$ 30873, ATCC 50677, $A T C C$ 50678, ATCC 50680, ATCC 50684, $A T C C$ 50686; $N=A T C C$ 50676; $O$ $=A T C C$ 50679; $P=A c / P H L / 23, A T C C$ 30868, ATCC 30873, ATCC 50676, ATCC 50677, ATCC 50678, ATCC 50680, ATCC 50684, ATCC 50686; 1 = Bausch \& Lomb Multi-Purpose Solution; 2 = Complete; $3=$ Optifree; $4=$ Optisoak; $5=$ Duracare; $6=$ Hydrocare; $7=$ Oxysept $1 ; 8=$ Total; $9=$ Transoak; $10=$ Transol . to contact lens solutions, $95 \%$ confidence intervals were constructed. In this analysis, overlapping confidence intervals indicate no significant difference, whereas those not overlapping indicate a significant difference.

\section{Results}

Information on the effect of different contact lens storage/soaking and disinfecting solutions on Acanthamoeba cysts is given in Figure 1, which represents the result of three repeated experiments. Each experiment gave reproducible results. All the control tubes containing amoeba saline plus cysts showed growth of organisms for all the contact times tested. The amoeba saline control was negative for growth, as were all the pure solutions tested.

Certain contact lens solutions killed trophozoites of strain ATCC 50676 faster than cysts of the same strain, but several solutions did not even kill trophozoites within the 7 day test period. Details of the results for trophozoites are not relevant to this paper and have not been included.

The most effective of the gas permeable and hard contact lens solutions tested on cysts of all strains was Transoak (Fig 1). Although viable cysts were still present at 2 hours, all had been inactivated by 4 hours.

Oxysept 1 was the most effective of the soft contact lens solutions tested. It killed cysts of strains ATCC 30873 (CCAP 1501/3d), ATCC 50677, ATCC 50679, and ATCC 50686 within 4 hours, whereas cysts of strains Ac/PHL/23, ATCC 30868 (CCAP 1501/2g), ATCC 50676, ATCC 50680, and ATCC 50684 were killed within 8 hours (Fig 1).

Table 2 Contact lens solutions tested

\begin{tabular}{|c|c|c|c|c|}
\hline Solution trade name & Manufacturer & Active ingredient $(s)^{*}$ & Preservative(s) & Type(s) of lens \\
\hline $\begin{array}{l}\text { Bausch \& Lomb Multi-Purpose } \\
\text { Solution } \dagger\end{array}$ & Bausch \& Lomb & $\begin{array}{l}\text { Polyaminopropyl biguanide (Dymed) } \\
(0.0005) \text {; } \\
\text { sodium borate }(1.20) ; \\
\text { sodium chloride }(4.9) ; \\
\text { poloxamine } 1107(10.00) \\
\text { boric acid }(6.40)\end{array}$ & $0.11 \%(\mathrm{wt} / \mathrm{vol})$ Disodium edetate & Soft \\
\hline Complete† & Allergan & Polyhexamethylene biguanide $(0.001)$ & & Soft \\
\hline Duracare $\ddagger$ & Allergan & $0.004 \%$ Polyvinyl alcohol & $\begin{array}{l}0.004 \% \text { Benzalkonium chloride; } \\
0.004 \% \text { Sodium edetate }\end{array}$ & Gas permeable \\
\hline $\begin{array}{l}\text { Hydrocare Cleaning/Soaking } \\
\text { Solution } \dagger\end{array}$ & Allergan & $\begin{array}{l}\text { Alkyl triethanol ammonium chloride } \\
(0.3)\end{array}$ & $0.002 \%$ Thiomersal & Soft \\
\hline Optifreet & Alcon & & $\begin{array}{l}0.001 \% \mathrm{~m} / \mathrm{v} \text { Polyquad } \\
\text { (polyquaternium- } 1 \text { ); } \\
0.05 \%(\mathrm{wt} / \mathrm{vol} \text { ) sodium edetate }\end{array}$ & Soft \\
\hline Optisoak† & Alcon & $\begin{array}{l}0.75 \text { g polyvinyl alcohol; } \\
0.005 \text { g polysorbate } 80 ; \\
0.65 \text { g hydroxyethyl cellulose } \\
\text { (all per } 100 \mathrm{ml} \text { ) } \\
\text { + sodium chloride } \\
\text { + sodium phosphates }\end{array}$ & $\begin{array}{l}\text { Polyquad, ie polyquaternium-1 } \\
0.005 \% \text { (wt/vol); } \\
\text { edetate sodium } 0.1 \%(\mathrm{wt} / \mathrm{vol})\end{array}$ & $\begin{array}{l}\text { Gas permeable and } \\
\text { hard }\end{array}$ \\
\hline Oxysept 15 & Allergan & Hydrogen peroxide (31.0) & & Soft \\
\hline Oxysept 1 Step 1 & Allergan & $3 \%$ Hydrogen peroxide & & Soft \\
\hline Total $\ddagger$ & Allergan & Polyvinyl alcohol (25.0) & $0.004 \%$ benzalkonium chloride & $\begin{array}{l}\text { Gas permeable and } \\
\text { hard }\end{array}$ \\
\hline Transoak $^{\star \star}$ & $\begin{array}{l}\text { Chauvin } \\
\text { Pharmaceuticals Ltd }\end{array}$ & $\begin{array}{l}0.01 \%(\mathrm{wt} / \mathrm{vol}) \text { benzalkonium } \\
\text { chloride }\end{array}$ & $0.2 \%(\mathrm{wt} / \mathrm{vol})$ disodium edetate & $\begin{array}{l}\text { Gas permeable and } \\
\text { hard }\end{array}$ \\
\hline Transol Wetting Solution†† & $\begin{array}{l}\text { Chauvin } \\
\text { Pharmaceuticals Ltd }\end{array}$ & $1 \mathrm{~g}$ polyvinyl alcohol $/ 50 \mathrm{ml}$ & $\begin{array}{l}0.004 \% \text { benzalkonium chloride; } \\
0.02 \%(\mathrm{wt} / \mathrm{vol}) \text { disodium edetate }\end{array}$ & $\begin{array}{l}\text { Gas permeable and } \\
\text { hard }\end{array}$ \\
\hline
\end{tabular}

*All data in parentheses are in $\mathrm{mg} / \mathrm{ml}$.

†Minimum stated soaking period for solution is 4 hours.

$\ddagger$ Minimum stated soaking period for solution is overnight (one manufacturer states that this is approximately 6-8 hours).

$\$$ Minimum stated soaking period for solution is 20 minutes.

TMinimum stated soaking period for solution is 2 hours.

${ }^{\star}$ Minimum soaking period not stated.

††Minimum soaking period is not stated as this is a wetting solution, not intended for soaking lenses.It was included as its constituents are similar to those of other soaking solutions tested, but it is manufactured by a different company. 
However, viable cysts of strain ATCC 50678 were still present after exposure to Oxysept 1 for 8 hours; but were non-viable after 3 days of exposure to this solution. Oxysept 1 Step, which is used for a period of 2 hours with a neutralising tablet, had no cysticidal effect within this time on any of the strains of Acanthamoeba tested.

Cysts of strains ATCC 30868 (CCAP 1501/ 2g) and ATCC 50679 were killed within 8 hours in Total but cysts of all other strains were still viable after exposure to this solution for 8 hours.

The time taken for the other solutions (Bausch \& Lomb Multi-Purpose, Complete, Duracare, Hydrocare, Optifree, Optisoak, and Transol) to kill cysts of all 10 strains was longer than 8 hours in most cases. Viable cysts were still present at 7 days in several cases (Fig 1).

STATISTICAL ANALYSIS

Statistically significant differences were detected between the following strains with regard to their susceptibility to contact lens solutions: Ac/PHL/23 v ATCC 30873; ATCC $30868 v$ ATCC 30873; ATCC 30868 v ATCC 50677; ATCC $30868 v$ ATCC 50680; ATCC $30868 v$ ATCC 50686. No significant differences were found between any of the other strains.

\section{Discussion}

A castellanii (ATCC 30868) was found to be the strain most commonly showing a statistically significant difference to other isolates in that less time was required for the contact lens solutions to kill this strain compared with the other isolates tested. It should be noted that Silvany et $a l^{27}$ found that complete inhibition of a strain of $A$ castellanii was generally achieved sooner than total inactivation of a strain designated as $A$ polyphaga.

Overall, we found that solutions for hard and gas permeable lenses were more effective against Acanthamoeba cysts than those for soft contact lenses. These results are similar to those of other authors. ${ }^{28}$

BENZALKONIUM CHLORIDE

Transoak $(0.01 \%$ benzalkonium chloride (BAK) and $0.2 \%$ disodium edetate) proved to be the most effective solution tested by us for anti-Acanthamoeba activity. It destroyed cysts of all strains within 4 hours. Lower concentrations of BAK do not appear to be as efficient, since Duracare, Total, and Transol, all of which contain $0.004 \%$ BAK and polyvinyl alcohol, generally took longer than 8 hours to kill cysts of the isolates tested. Our results with $0.004 \%$ BAK are similar to those of other authors. ${ }^{14} 29$ However, Silvany et $a l,{ }^{27}$ using the same strain of $A$ castellanii (ATCC 30868/CCAP 1501/2g) as Penley et $a l^{14}$ as well as a strain of $A$ polyphaga, found that $0.004 \%$ BAK was effective against cysts and trophozoites within 1 hour. A $0.003 \%$ BAK solution did not inactivate cysts of Acanthamoeba within the testing time. ${ }^{28} 30$

Higher levels $(0.01 \%$ to $4 \%)$ of BAK are known to cause significant corneal toxicity, but few effects have been noted at the concentration of $0.003 \%$, which is that used in several solutions for rigid gas permeable contact lenses. There is a progressive increase in damage at concentrations between $0.001 \%$ and $0.01 \%$, as determined by scanning electron microscopy. ${ }^{31} 32$ In addition, $0.01 \%$ BAK causes instability of the tear film, ${ }^{33}$ which makes the advisability of having all in one solutions questionable. ${ }^{34}$ BAK at a concentration of $0.01 \%$ should only be considered for use as an effective disinfecting solution for Acanthamoeba provided that this solution is not used for insertion of lenses into the eye and that binding of the BAK to the contact lenses does not occur, enabling it to be washed off by a wetting solution before the contact lens is inserted into the eye.

HYDROGEN PEROXIDE

Hydrogen peroxide disinfection has been thought to be a predisposing factor for Acanthamoeba keratitis. ${ }^{35}$ Different strains of Acanthamoeba tested by us show varying susceptibilities to this solution. Cysts of some strains were killed within 4 hours while viable cysts of other strains were still present at 8 hours. Our results with 3\% hydrogen peroxide on strain Ac/PHL/23 (SHI) are similar to those of Kilvington ${ }^{36}$ for this strain. The findings of different researchers with regard to the cysticidal activity of $3 \%$ hydrogen peroxide vary according to the strain of Acanthamoeba. ${ }^{1011} 131527-293738$ The taxonomic identity of the various isolates is "academic", however. What is important from a practical point of view is to know what will kill the most resistant strain of Acanthamoeba which might be present-for example, in a lens case, irrespective of its genetic relation. Davies et $a l^{13}$ found that whereas AOSept (3\% hydrogen peroxide with $0.85 \%$ sodium chloride) did not work, Oxysept (3\% hydrogen peroxide) had measurable anti-acanthamoebic activity. The authors suggested that this is possibly due to the difference in $\mathrm{pH}$ between the two solutions or to the presence of a neutralising catalytic disc in AOSept which renders the solution ineffective against Acanthamoeba. The presence of different stabilising ingredients may also result in slightly different amoebicidal effects for different products containing the same concentration of hydrogen peroxide. ${ }^{29}$

We found that Oxysept 1 Step (3\% hydrogen peroxide) was totally ineffective for inactivating cysts of the strains of Acanthamoeba tested. It should be noted that using a one step hydrogen peroxide system, Bilgin et $a l^{\beta 9}$ only achieved a $55 \%$ rate of disinfection for Pseudomonas sp, although $100 \%$ for other bacteria.

\section{ALKYL TRIETHANOL AMMONIUM CHLORIDE}

Hydrocare $(0.3 \mathrm{mg} / \mathrm{ml}$ alkyl triethanol ammonium chloride) was ineffective against cysts of the strains of Acanthamoeba tested by us. The results of some previous studies are similar. ${ }^{10} 111428$ In contrast, Davies et $a l^{13}$ and Silvany et $a l^{27}$ found that Hydrocare killed cysts of Acanthamoeba within 4 hours. 
POLYAMINOPROPYL BIGUANIDE

Polyaminopropyl biguanide (PAPB), 0.0005 $\mathrm{mg} / \mathrm{ml}$, (Bausch \& Lomb Multi-Purpose solution) was found by us to be totally ineffective against Acanthamoeba cysts. Viable cysts of all the strains of amoebae tested were present after 7 days of exposure to this solution. The results of other authors are in agreement with ours. ${ }^{13} 14282937$ However, Silvany et $a l^{27}$ determined that although a $0.00005 \%$ PAPB solution was effective at 12 hours against $A$ castellanii (strain ATCC 30868), it was not effective against $A$ polyphaga cysts even at 24 hours. At a higher concentration-namely, $0.0015 \%$, PAPB was found to be ineffective against Acanthamoeba cysts by Connor et al ${ }^{28}$ and Hugo et al. ${ }^{30}$ It is relevant that a $0.0015 \%$ PAPB solution caused increased corneal staining by fluorescein, indicating sloughing of cells, compared with control eyes. ${ }^{31}$

POLYHEXAMETHYLENE BIGUANIDE

Complete $(0.001 \mathrm{mg} / \mathrm{ml}$ polyhexamethylene biguanide (PHMB)) did not inactivate cysts of any of the strains of Acanthamoeba tested. Viable cysts were detected in all experiments after 7 days of exposure to the solution. At a concentration of $0.00005 \%$, PHMB has previously been shown to be ineffective against Acanthamoeba cysts. ${ }^{27}{ }^{36}$ However, PHMB is useful at higher concentrations for treating Acanthamoeba keratitis. ${ }^{4041}$

POLYQUAD

Optifree (0.001\% polyquad) and Optisoak $(0.005 \%$ polyquad $)$ did not kill the strains of Acanthamoeba that we worked with. Cysts of all strains were found to be viable after 7 days of exposure to these solutions. The absence of any marked cysticidal effect of $0.001 \%$ polyquad against cysts of Acanthamoeba has also been demonstrated by other authors. ${ }^{13} 1427-29$

\section{GENERAL COMMENTS}

Over half of the solutions examined by Richardson et $a l^{25}$ contained less than $90 \%$ of the stated preservative content. In the case of thiomersal, only $2 / 15$ solutions were within the acceptable limits of $90-110 \%$ of declared preservative concentration; and one solution contained $170 \%$ of the stated amount. This finding is highly significant as it could explain why researchers obtain varying results - that is, the actual concentration of active ingredient may not always be that stated as being present. BAK shows the least interaction with plastics and loss of this preservative from these systems is probably not biologically significant. ${ }^{25}$ This retention of a high level of activity in the storage container possibly enhances the consistent effectiveness of BAK as present in Transoak, against cysts of different strains of Acanthamoeba. Richardson et $a l^{55}$ commented that sorption in storage containers appeared to occur with thiomersal and chlorbutol in contrast with BAK and chlorhexidine gluconate, which are known to interact mainly by a surface adsorption process. The extent of the interactions was found to be dependent upon the type of plastic material of which the container is made.

We assessed viability versus non-viability of cysts. Even though it could be of interest to ascertain at what rate amoebae are killed off, ${ }^{13}$ we feel that the importance lies in knowing at what stage all amoebae are non-viable, because even one viable cyst could potentially cause keratitis should the eye be invaded.

Discrepancies in results reported by various authors could possibly also be due to the age of the cysts. Our visual observations indicate that cyst walls become thicker as the cyst ages. It might be more difficult for chemicals to penetrate older and thicker walls, making the cysts more resistant to inactivation. Brandt et $a l^{11}$ grew amoebae for 4-6 weeks on agar plates. These cysts were much older than those used by other authors - namely, cysts used within 7 days. ${ }^{13}$ The time taken for encystment to occur is speeded up when an encystment medium is used (as we did), in which case the process occurs within 20 hours. Within 48 hours, encystment is greater than $90 \% .^{13}{ }^{23}$

Yet another potential problem that could occur is if the container in which the experiment is conducted is agitated so that cysts or trophozoites come out of the suspension and adhere to the side of the tube or the lid. The exposed amoeba would no longer be in contact with the solution for the full experimental period. At the time of washing and plating, the organism could be washed back into the solution, subsequently giving rise to a false positive result when the amoeba multiplies. Furthermore, cysts in the middle of any pellet might, likewise, not be exposed to the solution for the full period of time. We accordingly used a sterile pipette to gently bubble amoebae through the solution so as to ensure that they were not left in a pellet at the bottom of the tube.

We also feel that it is important for the experiment to be carried out in the dark as the potency of the disinfectants may be affected by light. Most lens cases and bottles containing solutions are made of coloured plastic which does not allow bright light through into the solutions.

The normal period of time for lenses to be disinfected would be overnight, while the wearer is sleeping. We would therefore consider a solution that kills amoebae within 5 hours to be safe for users. The minimum stated soaking time for the solutions we tested, when recommended by the manufacturers (Table 2), was in no instance sufficient for inactivating cysts of the strains of Acanthamoeba tested by us. It is, therefore, very important that manufacturers of disinfectants are made aware of the time required to kill Acanthamoeba, so that users can be advised as to the appropriate time for soaking contact lenses in the solution.

Compliance with manufacturers' recommendations concerning contact lens disinfection appears, in general, to be unsatisfactory. Recommendations by attending optometrists and ophthalmologists should be adhered to. Seal and $\mathrm{Hay}^{6}$ have suggested that contact lens wearer compliance could be improved by the 
development of a compact, single use disposable disinfection system.

The wiping of contact lenses with a daily cleaner can perhaps also be considered as part of the disinfection procedure which, owing to the physical action of rubbing the lenses, may help to dislodge some amoebic cysts or trophozoites from contact lenses. Our experience in the laboratory, however, is that amoebae tend to attach so firmly to plastic culture dishes and non-siliconised glassware, that vigorous rubbing would be required to remove all the Acanthamoeba cysts that might be adhering to the contact lenses or lens case. John ${ }^{42}$ and John et $a l^{43}$ have shown that both cysts and trophozoites stick to extended wear soft contact lenses and that the process of washing the lenses did not remove all the trophozoites and cysts from the contact lens surface. Consequently, the wearer needs to ensure that any amoebae introduced into the contact lens storage case or adhering to contact lenses would be killed by the soaking solution used.

Our studies have shown that the killing time for cysts of the African and UK isolates of Acanthamoeba that we studied is, in general, similar. Guidelines for contact lens solution usage suggested in the UK would therefore be appropriate for the increasing number of travellers who visit South Africa as tourists or on business as well as for local residents in South Africa.

This work was supported by the South African Medical Research Council and the Medical Faculty Research Endowment Fund of the University of the Witwatersrand, Johannesburg, South Africa.

We thank $\mathrm{S}$ Kilvington and DC Warhurst for providing strains of Acanthamoeba from England, and Mrs JMG van Deventer for technical assistance.

1 Seal D, Stapleton F, Dart J. Possible environmental sources of Acanthamoeba spp in contact lens wearers. $\mathrm{Br} \mathcal{F}$ Ophthalmol 1992;76:424-7.

2 Kilvington S, Larkin DFP, White DG, et al. Laboratory investigation of Acanthamoeba keratitis. 7 Clin Microbiol 1990;28:2722-5.

3 Markus MB, Niszl IA. Contact lens warning in relation to Acanthamoeba. S Afr Med f 1990;78:645.

4 Kingston D, Warhurst DC. Isolation of amoebae from the air $f$ Med Microbiol $1969 ; 2: 27-36$.

5 Bottone EJ, Madayag RM, Qureshi MN. Acanthamoeba keratitis: synergy between amebic and bacterial cocontakeratitis: synergy between amebic and bacterial coconta-
minants in contact lens care systems as a prelude to infec-

6 Seal DV, Hay J. Contact lens disinfection and Acanthamoeba: Problems and practicalities. Pharm f 1992; 248:717-19.

7 Larkin DFP, Kilvington S, Easty DL. Contamination of contact lens storage cases by Acanthamoeba and bacteria Br f Ophthalmol 1990;74:133-5.

8 Clark BJ, Harkins LS, Munro FA, et al. Microbial contamination of cases used for storing contact lenses. F Infection 1994;28:293-304.

9 Stehr-Green JK, Bailey TM, Brandt FH, et al. Acanthamoeba keratitis in soft contact lens wearers. A case-control study. $7 A M A$ 1987;258:57- 60.

10 Ludwig IH, Meisler DM, Rutherford I, et al. Susceptibility of Acanthamoeba to soft contact lens disinfection systems. Invest Ophthalmol Vis Sci 1986;27:626-8.

11 Brandt FH, Ware DA, Visvesvara GS. Viability of Acanthamoeba cysts in ophthalmic solutions. Appl Environ Microbiol 1989;55:1144-6.

12 Davies DJG, Anthony Y, Meakin BJ, et al. AntiAcanthamoeba activity of chlorhexidine and hydrogen peroxide. Trans Br Contact Lens Assoc Int Conf 1988:60-2.

13 Davies DJG, Anthony Y, Meakin BJ, et al. Evaluation of the anti-acanthamoebal activity of five contact lens disinfectants. Int Contact Lens Clin 1990;17:14-20.
14 Penley CA, Willis SW, Sickler SG. Comparative antimicrobial efficacy of soft and rigid gas permeable contact lens solutions against Acanthamoeba. CLAO f 1989;15:25760.

15 Lindquist TD, Doughman DJ, Rubenstein JB, et al. Acanthamoeba-contaminated hydrogel contact lenses. Cornea 1988;7:300-3.

16 Meisler DM, Rutherford I. Acanthamoeba and disinfection of soft contact lenses. Rev Infect Dis 1991;13(Suppl 5):S410-12.

17 Kilvington S, Beeching JR, White DG. Differentiation of Acanthamoeba strains from infected corneas and the environment by using restriction endonuclease digestion of whole-cell DNA. f Clin Microbiol 1991;29:310-14.

18 Nagington J, Watson PG, Playfair TJ, et al. Amoebic infection in the eye. Lancet 1974;ii:1537-40.

19 Badenoch PR, Adams M, Coster DJ. Corneal virulence, cytopathic effect on human keratocytes and genetic character ization of Acanthamoeba. Int $\mathcal{F}$ Parasitol 1995;25: 229-39.

20 Page FC. A new key to freshwater and soil Gymnamoebae with instructions for culture. Ambleside: Freshwater Biological Association, 1988

21 Visvesvara GS. Classification of Acanthamoeba. Rev Infect Dis 1991;13(Suppl 5):S369-72.

22 Niszl IA, Markus MB, van Deventer JMG. Anti-Mastigina activities of eight contact lens solutions. Antimicrob Agents Chemother 1995;39:2364-6.

23 Neff RJ, Ray SA, Benton WF, Wilborn M. In: Prescott DM, ed. Methods in cell physiology. Vol 1. New York: Academic Press, 1964:55-83.

24 De Jonckheere J, Van De Voorde H. Differences in destruction of cysts of pathogenic and nonpathogenic Naegleria and Acanthamoeba by chlorine. Appl Environ Microbiol 1976;31:294-7.

25 Richardson NE, Davies DJG, Meakin BJ, et al. Loss of antibacterial preservatives from contact lens solutions during storage. F Pharm Pharmacol 1977;29:717-22.

26 Osato MS, Robinson NM, Wilhelmus KR, et al. In vitro evaluation of antimicrobial compounds for cysticidal activity against Acanthamoeba. Rev Infect Dis 1991;13(Suppl 5):S431-5.

27 Silvany RE, Dougherty JM, McCulley JP, et al. The effect of currently available contact lens disinfection systems on Acanthamoeba castellanii and Acanthamoeba polyphaga. Ophthalmology 1990;97:286-90.

28 Connor CG, Hopkins SL, Salisbury RD. Effectivity of contact lens disinfection systems against Acanthamoeba culbertsoni. Optom Vis Sci 1991;68:138-41.

29 Zanetti S, Fiori PL, Pinna A, et al. Susceptibility of Acanthamoeba castellanii to contact lens disinfecting solutions. Antimicrob Agents Chemother 1995;39:1596-8.

30 Hugo ER, McLaughlin WR, Oh K, et al. Quantitative enumeration of Acanthamoeba for evaluation of cyst inactivafion in contact lens care solutions. Invest Ophthalmol Vis Sci 1991;32:655-7.

31 Begley CG, Weirich B, Benak J, et al. Effects of rigid gas permeable contact lens solutions on the human corneal epithelium. Optom Vis Sci 1992;69:347-53.

32 Burstein NL. Preservative cytotoxic threshold for benzalkonium chloride and chlorhexidine digluconate in cat and rabbit corneas. Invest Ophthalmol Vis Sci 1980;19:308-13.

33 Wilson WS, Duncan AJ, Jay JL. Effect of benzalkonium chloride on the stability of the precorneal tear film in rabbit and man. Br f Ophthalmol 1975;59:667-9.

34 Phillips AJ, Stone J. Contact lenses. 3rd ed. London: Butterworths, 1989:131-85.

35 Cohen EJ, Parlato CJ, Arentsen JJ, et al. Medical and surgical treatment of Acanthamoeba keratitis. Am $\mathcal{F}$ Ophthalmol 1987;103:615-25

36 Kilvington S. Activity of biocide chemicals and contact lens disinfectants on pathogenic free-living amoebae. Int Biodeter 1990;26:127-38.

37 Connor CG, Blocker Y, Pitts DG. Acanthamoeba culbertsoni and contact lens disinfection systems. Optom Vis Sci 1989;66:690-3.

38 Silvany RE, Wood TS, Bowman RW, et al. The effect of preservatives in contact lens solutions on two species of Acanthamoeba. Invest Ophthalmol Vis Sci 1987;28(Suppl):371.

39 Bilgin LK, Manav G, Tutkun TT, et al. Efficacy of a one-step hydrogen peroxide system for disinfection of soft contact lenses. CLAO F 1993;19:50-2.

40 Larkin DFP, Kilvington S, Dart JKG. Treatment of Acanthamoeba keratitis with polyhexamethylene biguanide. Ophthalmology 1992;99:185-91.

41 Niszl IA, Markus MB. Treatment of Acanthamoeba keratitis. S Afr Med 7 1996;86:566.

42 John T. Interactions of bacteria and amoebae with ocular biomaterials. Cells and Materials 1991;1:129-39.

43 John T, Desai D, Sahm D. Adherence of Acanthamoeba castellanii cysts and trophozoites to unworn soft contact lenses. Am f Ophthalmol 1989;108:658-64. 